\title{
EFFECTS OF PHYSICAL EXERCISE ON A MULTIPAROUS PATIENT IN THE PREPARTUM PERIOD. CASE STUDY
}

\author{
Horia CROITORU ${ }^{1}$, Silvia TEODORESCU ${ }^{1}$, Carmen ENE-VOICULESCU ${ }^{2}$ \\ ${ }^{1}$ National University of Physical Education and Sport, Faculty of Physical Education and Sport, Bucharest, \\ Romania \\ 2 “Ovidius” University, Faculty of Physical Education and Sport, Constanța, Romania \\ *Corresponding author: croitoru.horia@yahoo.com
}

https://doi.org/10.35189/dpeskj.2020.59.2.3

\begin{abstract}
Starting from the premise that physical exercise in the prepartum period is associated with benefits for both the mother and foetus, the purpose of this paper is to highlight the evolution of gestational periods of a patient during two different pregnancies. The proposed case study presents a comparative analysis of the prepartum and postpartum periods of a 33-year-old patient, considering that she had a sedentary lifestyle during the first pregnancy, while in the prepartum period related to the second pregnancy, the patient actively participated in a physical exercise programme adjusted to each trimester of pregnancy. The main objectives of the training programme focused on correcting postural defects caused by the first pregnancy, maintaining blood glucose levels within normal limits and preventing the recurrence of gestational diabetes, but also reducing blood pressure fluctuations. Patient evaluation took place 12 weeks after both the first and second childbirth and consisted in assessing her quality of life during the two gestational periods through questionnaires, anthropometric measurements and specific fitness tests. The obtained results showed that, following the practice of physical exercise during the gestational period of the second pregnancy, the patient managed to maintain her blood glucose levels within normal limits without suffering this time from gestational diabetes (a pre-existing condition at her first pregnancy) and no longer had either fluctuations in blood pressure or lumbar pain caused by the exacerbation of her lumbar lordosis during the first gestational period.
\end{abstract}

Keywords: prepartum, postpartum, gestational diabetes, multiparous patient.

\section{Introduction}

\section{Physical activity during pregnancy}

Pregnancy is a special condition in which a woman's body undergoes multiple transformations during the nine months. Among the important changes that occur in the body, we highlight: accelerated heart rate and low blood pressure due to the increase in blood flow; enlarged blood vessels and increased pressure exerted by the constantly growing uterus on the arteries, which leads to slowing down the speed of blood to the heart; an increased number of breaths per minute due to the pressure exerted by the enlarged abdomen on the diaphragm - especially in the last trimester of pregnancy, when the size and weight of the uterus put additional pressure on the back muscles; exacerbated curves of the spine, which leads to the emergence of lumbar pain; increased body weight; increased water retention in the body along with venous stasis, which will cause oedema in the lower limbs and the sensation of tired legs. To get through this period more easily, women are encouraged to practice physical exercise aimed to ensure good mental tone, optimal weight gain and better aerobic condition. In most pregnancies, mild-to-moderate physical activity is beneficial for the mother and will not have undesirable effects on the unborn baby. 
On the opposite side, sedentary lifestyle is considered the major cause of obesity, diabetes, breast cancer and other chronic conditions. Studies published by the Sedentary Behavior Research Network and confirmed by the World Health Organisation and the American College of Obstetrics and Gynecology (ACOG, 2020) report a growing number of pregnant women who adopt a sedentary lifestyle in both the prepartum and postpartum periods. The literature data on women's behaviour in the gestational period attest that over $50 \%$ of the diurnal period is characterised by a sedentary lifestyle (Fazzi et al., 2017). The authors of another study conducted on a group of 70 pregnant women in Montreal (Canada) analysed the sedentary behaviour of subjects using an accelerometer worn for 3 days in different periods of pregnancy (weeks 16-18, weeks 24-26, weeks 32-34) and correlated the data with those obtained after applying a questionnaire aimed to highlight the level of perceived stress during monitoring (Sinclair et al., 2019). The results have shown that there is a significant correlation between the level of psychosocial stress and the adoption of a sedentary lifestyle during pregnancy.

The gestational period is the optimal time to implement physical exercise in daily life in order to obtain structural and functional benefits for the body in the short term, but also in the long term. Given the declining proportion of physically active young women and the higher incidence of cardiovascular disease and obesity among the same category of people, increasing the level of physical activity is essential in combatting sedentary lifestyle and related conditions. This is confirmed by the data presented by Dipietro et al. (2019), who demonstrate that moderate-intensity physical activity has reduced the risk of excessive increase in gestational weight, gestational diabetes and postpartum depression symptoms. However, the aforementioned authors have failed to determine whether these issues vary according to age, race/ ethnicity, socioeconomic status or the values of body weight before pregnancy.

Extending the research activity to the modifications that occur in a pregnant woman's body, therefore beyond the simple evolution of pregnancy, it has been demonstrated that physical exercise in the prepartum period is associated with benefits for both the mother and foetus. A comprehensive study conducted in nine states (Australia, Canada, Denmark, France, Japan, Norway, Spain, the United Kingdom and the Unites States) identified 11 guidelines to be considered when implementing physical exercise programmes during the prepartum period. The guidelines developed following the studies carried out recommend practicing moderate physical activity with a frequency and duration adapted to the needs of each subject, as well as avoiding activities that might present a risk of fall, trauma or injury (Evenson et al., 2014).

\section{Methodology and Results}

\section{Case study}

We present the case of a multiparous young woman (G.L.) aged 33 years, who underwent caesarean sections at both her first and second childbirth. The first delivery took place when she was 28 years old, and the second delivery, at the age of 31 years. 
The young woman lives in an urban area, is a graduate of higher education, and the only period when she was employed was during her university studies, because she has become a housewife after graduation. It should be mentioned that, until the moment of conception and during the first pregnancy, the patient did not practice any form of physical exercise, declaring herself a sedentary person. The only physical activities in which she took part were everyday activities such as housework, daily shopping and occasional walks.

Following clinical examinations performed after the onset of first pregnancy (May 2016), the young woman was declared clinically healthy, with no other associated conditions that might have affected the evolution of pregnancy. The gestational period was clinically normal, except that the patient was diagnosed with gestational diabetes that she kept under control through supervised diet and without practicing physical exercise. Pregnancy ended with a caesarean section upon recommendation of the gynaecologist (February 2016).

Twelve weeks after the caesarean delivery, also upon recommendation of the gynaecologist and encouraged by her family, the young woman wanted to start a physical exercise programme dedicated to the postpartum period. This programme began (in May 2016) with the case history during which anthropometric measurements were performed, the initial fitness level was determined by specific tests, and the perceived evolution of pregnancy was assessed using a questionnaire aimed to identify body changes as perceived by the patient in the gestational period.

As a result of anthropometric measurements, the following data were obtained: height: 160 cm; weight: $60 \mathrm{~kg}$; bust circumference: $80 \mathrm{~cm}$; arm circumference: $26 \mathrm{~cm}$; upper-abdomen circumference: $75 \mathrm{~cm}$; lower-abdomen circumference: $87 \mathrm{~cm}$; pelvis circumference: $96 \mathrm{~cm}$; thigh circumference: $56 \mathrm{~cm}$.

Evolution and perceived changes during pregnancy:

- the patient suffered from gestational diabetes diagnosed in the 25 th week of pregnancy;

- type of delivery: caesarean section (under medical advice);

- no complications were reported during childbirth;

- blood glucose level returned to normal parameters after delivery (glucose tolerance test at 8 weeks after childbirth);

- the patient complained of fluctuations in blood pressure without being diagnosed with high/low blood pressure;

- the patient felt discomfort in the lumbar area when maintaining the standing position for a longer period (pain was generated by the exacerbation of her lumbar lordosis, but also by a pelvic deformity in the frontal plane);

- discomfort in the lumbar area during pregnancy also persisted in the postpartum period;

- in the postpartum period, the patient complained of limited lumbar flexibility.

Basal fitness level testing

○ Flamingo Balance Test: 18 attempts (rating: poor);

○ Trunk flexion on the pelvis from sitting: $-6 \mathrm{~cm}$ (rating: poor);

○ Standing long jump: $125 \mathrm{~cm}$ (rating: poor);

○ Sit-ups: 10 repetitions (rating: poor); 
- Shuttle run: level 3; 7 laps/level (rating: poor).

Following the assessment of postpartum fitness level by applying the 5 specific tests, it is noted that the patient has been rated "poor" in all 5 tests.

\section{Conclusions after the postpartum assessment}

Following the gynaecologist's recommendations and after the assessment of basal fitness level, it was found that the young mother, although she had poor scores in all tests, could practice physical exercise because she had been able to perform all 5 tests. After assessing and interpreting the data obtained from the questionnaire applied to the patient, we could notice her desire to attend the postpartum physical rehabilitation programme. A favourable family environment was created for the young mother to take part in the postpartum rehabilitation programme. Her income level was above the population average, so she could bear the costs generated by her participation in the programme (appropriate equipment, club access subscription, etc).

\section{Objectives and periodisation of physical exercise in the postpartum period}

After analysing the data obtained from the initial assessment, we developed the following main objectives:

1. Improving postural imbalances in the spine;

2. Regaining joint mobility and muscle elasticity;

3. Reducing blood glucose fluctuations and insulin resistance;

4. Developing the upper- and lower-body muscles;

5. Increasing the anaerobic and aerobic exercise capacity;

The periodisation of physical exercise in the postpartum period had 3 stages that were distributed as follows:

a) Stage I - Anatomical adaptation for 12 weeks

At this stage, the training was performed in the $3+1$ system ( 3 weeks of progressive loading +1 week of recovery). A week included 3 workouts as follows:

- 2 sessions dedicated to general strength;

- 1 session for the development of aerobic endurance and mobility.

b) Stages II and III - toning/ myofibrillar hypertrophy

The difference between the two stages is given by the number of training sessions per week and the distribution of targeted muscle groups by day.

Stage II consisted of 4 training sessions per week, which were distributed as follows:

- a session for the development of lower-body muscles;

- a session for the development of aerobic endurance and mobility;

- a session for the development of upper-body muscles;

- a session for the development of aerobic endurance and mobility.

Stage III included 5 training sessions per week, which were distributed as follows:

- a session for the development of upper-body muscles, with an emphasis on pectoral, deltoid and triceps muscles (A1 - push);

- a session for the development of aerobic endurance and mobility (A2); 
- a session for the development of lower-body muscles (A3 - legs);

- a session for the development of aerobic endurance and mobility (A2);

- a session for the development of upper-body muscles, with an emphasis on all dorsal and biceps muscles (A4 - pull).

At the end of stage III, the young woman was again assessed in terms of both fitness level and anthropometric measurement, and the results were as follows (Figure 1):

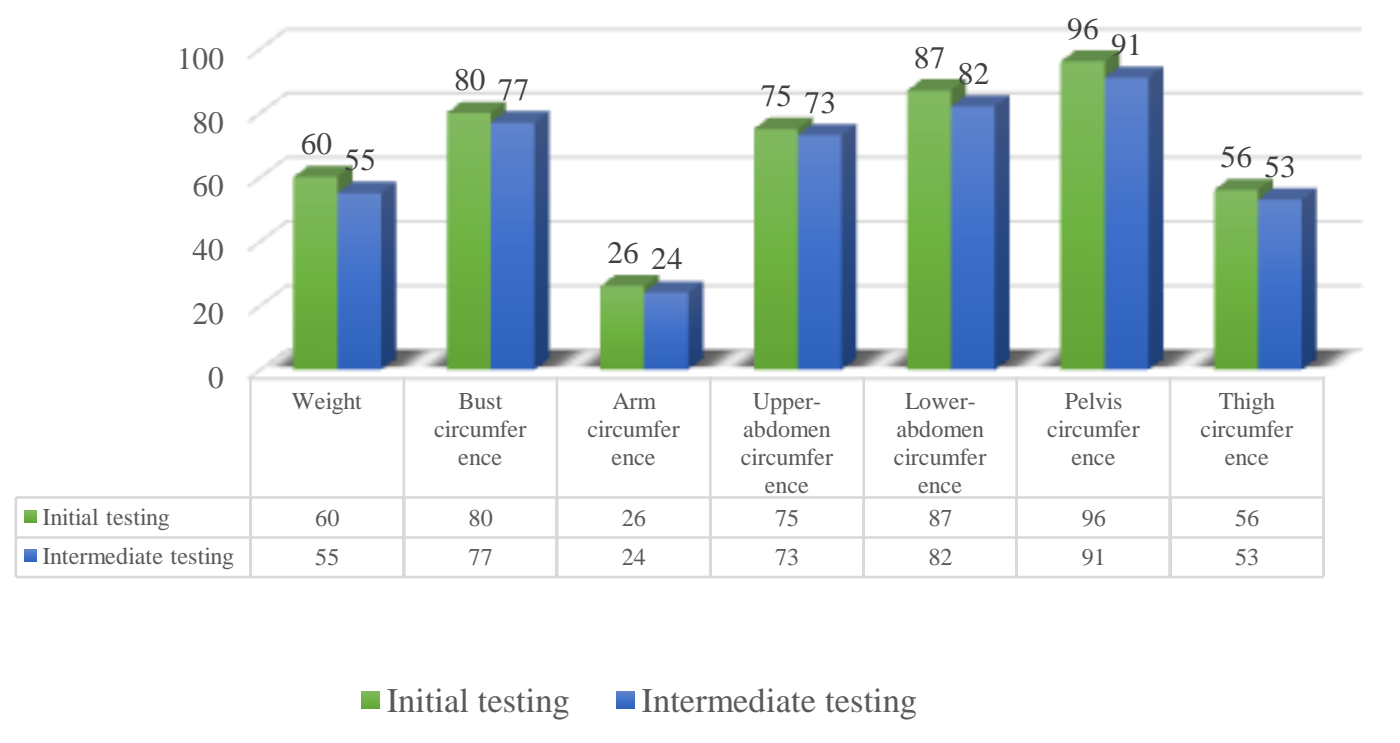

Figure 1. Comparative values of anthropometric measurements before and after performing the physical exercise programme

Results for the intermediate assessment of fitness level

○ Flamingo Balance Test: 14 attempts (rating: good);

○ Trunk flexion on the pelvis from sitting: $-2 \mathrm{~cm}$ (rating: good);

○ Standing long jump: $160 \mathrm{~cm}$ (rating: good);

○ Sit-ups: 21 repetitions (rating: very good);

○ Shuttle run: level 5; 8 laps/level (rating: good).

Following the intermediate assessment of the postpartum fitness level by applying specific tests, it is noted that the patient has obtained 4 "good" scores and a "very good" score in the tests performed. Thus, we can conclude that all monitored motor and functional parameters have been improved during the postpartum physical exercise programme.

At the end of April 2017, after a specialist consultation, the physician confirmed that the young woman was pregnant for the second time and she was advised not to completely stop physical activity for the next 10-12 weeks, but to reduce it so that her heart rate does not exceed 110-120 beats per minute, to avoid exerting high pressure on the abdominal and pelvic muscles and to exclude all physical activities that might present a risk of fall or injury. 


\section{Periodisation of physical exercise in the prepartum period}

Physical training in the prepartum period was spread out over 36 weeks (but could be suspended at any time if the physician recommended stopping physical activity or the young woman no longer wanted to continue the established training programme). With the beginning of the 3rd trimester of pregnancy, the exercise intensity and muscle work were reduced: the training sessions for muscle toning had a global character in order to avoid overusing the upper- or lower- body muscles; mobility and endurance workouts used mobility exercises in a larger proportion and a much reduced intensity of endurance exercise (Table 1).

To note that a permanent collaboration relationship was kept between patient, gynaecologist and the prepartum training coordinator.

Periodisation of physical exercise in the prepartum period (macrocycle):

- Stage I - Anatomical adaptation

- Weeks 0-6: Break;

- Weeks 7-12 (2 sessions/ week): swimming and mobility;

- Weeks 13-16 (3 sessions/ week): development of upper-body muscles; endurance + mobility; development of lower-body muscles;

- $\quad$ Stage II

- Weeks 17-20 (3 sessions/ week): development of upper-body muscles; endurance + mobility; development of lower-body muscles;

- Weeks 21-24 (3 sessions/ week): development of upper-body muscles; endurance + mobility; development of lower-body muscles;

- $\quad$ Stage III

- Weeks 25-28 (2 sessions/ week): overall training for muscle development; mobility + endurance;

- Weeks 29-32 (2 sessions/ week): overall training for muscle development; mobility + endurance;

- Weeks 33-36 (2 sessions/ week): overall training for muscle development; mobility + endurance.

The young woman took part in this programme until the 34th week of pregnancy, being permanently in touch with her gynaecologist. The option to temporarily leave the programme belonged to the patient, without receiving this advice from her gynaecologist. Throughout the programme, her pregnancy had normal evolution, which is why the specialist physician encouraged the young woman to persevere in practicing physical exercise.

After leaving the prepartum training programme, the patient continued to have a normal daily physical activity, without encountering difficulties in carrying out domestic and leisure activities. 
Comparative results obtained from the case history conducted after 12 weeks of postpartum for both the first and second pregnancies (Figure 2).

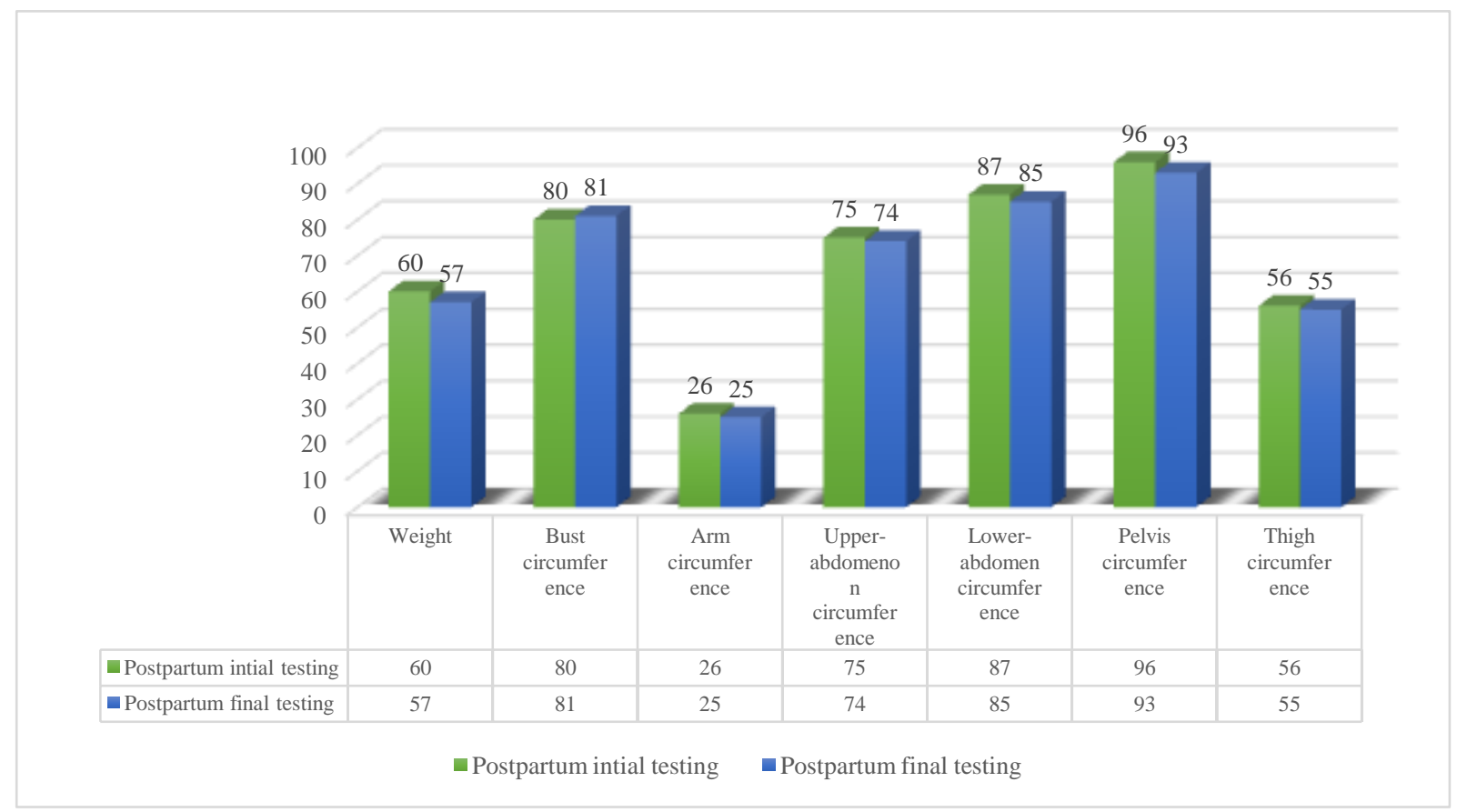

Figure 2. Comparative values of anthropometric measurements in the postpartum periods related to the two pregnancies

The results obtained after applying the postpartum questionnaire are shown in Table 1, and the patient's fitness levels in the postpartum periods related to the two pregnancies are shown in Table 2.

Table 1. Results obtained after applying the postpartum questionnaire

\begin{tabular}{ll}
\hline Perceived changes during the first pregnancy & Perceived changes during the second pregnancy \\
\hline - gestational diabetes & $\bullet$ optimal blood glucose level \\
- blood pressure fluctuations & $\bullet$ normal blood pressure (without fluctuations) \\
- discomfort in the lumbar area (exacerbated lumbar & $\bullet$ no pain was reported in the lumbar area \\
lordosis) & \\
- discomfort in the lumbar area also persisting in the & $\begin{array}{l}\text { - no postpartum pain was reported in the } \\
\text { postpartum period }\end{array}$ \\
- limited upper-body mobility (anterior trunk flexion) & \begin{tabular}{l} 
- regained upper-body mobility \\
\hline
\end{tabular}
\end{tabular}

Table 2. Patient's fitness levels in the postpartum periods related to the two pregnancies

\begin{tabular}{lcc}
\hline \multicolumn{1}{c}{ Tests } & $\begin{array}{c}\text { Fitness level testing after the first } \\
\text { delivery }\end{array}$ & $\begin{array}{c}\text { Fitness level testing after the } \\
\text { second delivery }\end{array}$ \\
\hline Flamingo Balance Test & 18 attempts & 15 attempts \\
Trunk flexion on the lower limbs & $-6 \mathrm{~cm}$ & $-1 \mathrm{~cm}$ \\
Standing long jump & $125 \mathrm{~cm}$ & $150 \mathrm{~cm}$ \\
Sit-ups & 10 repetitions & 15 repetitions \\
Shuttle run & Level 3 & Level 4 \\
& Poor & Good \\
\hline
\end{tabular}




\section{Discussion}

The evolution of mother's body and its morpho-functional changes in the prepartum period are the result of adaptation to both the needs of foetal development in the uteroplacental system and the readjustment of the main control centre, the hypothalamus, under the action of progesterone (Hytten, 1968). Physical exercise aims to facilitate these changes but should intervene only in case of non-pathological symptoms.

An important role in body evolution throughout the gestational period is played by the osteo-myo-arthrokinetic system. In the gestational period, this system undergoes functional and structural changes in all three components that make it up (bones, joints, muscles). The bones undergo a decrease in calcium concomitantly with an increase in elasticity (Ireland \& Ott, 2000). The joints gain an increased degree of mobility especially in the spine and pubic symphysis, and as a result of associating this with the increased weight of patient's body, changes in the statics may occur by exacerbating postural imbalances such as lumbar lordosis or improper positioning of scapulohumeral and pelvic girdles. About 50\% of pregnant women report discomfort, low back pain and pelvic girdle pain, and 25\% state that these symptoms persist even one year after childbirth (Gutke et al., 2006).

Low back pain (LBP) is located between the 12th vertebra and gluteal fold, and pelvic girdle pain (PGP) is felt between the posterior iliac crest and gluteal fold. Although described separately, they are analysed as the aggregation of structural and functional changes perceived during a gestational period (Gutke et al., 2006).

Under the action of progesterone and due to the lack of physical exercise to maintain muscle tone, muscles in general, but particularly abdominal muscles become hypotonic and therefore unable to keep the pregnant uterus in a normal position, allowing the formation of a uterus pouch concomitantly with an increasingly pronounced abdominal distension (Ireland \& Ott, 2000).

The American College of Obstetrics and Gynecology (ACOG) encourages pregnant women who were active before the gestational period and whose pregnancy is not at risk to maintain most of the physical activities they used to perform until the time of conception or to practice at least 150 minutes of physical activity per week. Instead, women who were physically inactive in the period preceding pregnancy are advised to gradually introduce light physical exercise into their daily routine (ACOG, 2020; da Silva et al., 2017).

The data presented by numerous systematic analyses reveal that practicing physical exercise during the gestational period can reduce the risk of weight gain in both the mother and foetus, decrease the incidence of gestational diabetes, gestational hypertension, preeclamsia, macrosomia, urinary incontinence and even depressive disorders (Davies \& Artal, 2018).

Following several studies that highlight sporadic episodes of foetal bradycardia during the practice of physical exercise, a statement has been made that uterine blood flow can be disrupted by physical exercise, which can lead to foetal hypoxia. However, research on this topic has demonstrated that the utero-placental system can compensate for transient changes in blood flow during physical exercise and there is no risk for the foetus even in women who were sedentary before pregnancy (Davenport et al., 2019). 
The exercise intensity is recommended to be adapted to the specific features and fitness levels of subjects, including easy-to-quantify physical activities. Recommendations for previously sedentary patients are related to practicing activities with an intensity ranging from low to moderate. Although a superior level of exercise intensity is not established for pregnancy, it is considered that pregnant women that have been active in the period before pregnancy can continue to practice physical activity with a higher intensity, for instance jogging, certain types of aerobic exercise, as well as interval physical exercise. In these cases, the exercise intensity can be monitored based on the perceived effort and the heart rate, allowing the pregnant woman to work at her own pace, which will voluntarily decrease with the evolution of the gestational period (ACOG, 2020).

Subjects practicing performance sports should be aware of a decrease in fitness levels with the evolution of pregnancy, even if there may be cases where an improvement in physical performance is possible, but only under specialised medical guidance (Evenson et al., 2014).

\section{Conclusion}

Similar to a chronic condition whose treatment does not consist in a surgical intervention or a short-term cure, sedentariness is a condition that is quickly spreading among all social classes and age categories, and cannot be combatted with rapid treatment, but only with ambition, determination and the long-term involvement of people in a wide range of attractive and pleasant physical activities.

The effects of sedentary lifestyle can be identified in any person that refuses to engage in any kind of physical activity, but mostly in people who are labelled as vulnerable in physical, mental and emotional terms.

Pregnant women are vulnerable both mentally and emotionally, but especially physically due to the dramatic reduction of physical activity during the gestational period, without being aware of the implications of this decision on the evolution and management of pregnancy. According to the literature, sedentary lifestyle can be a silent enemy that can leave a strong mark on both the health status of the future mother and the foetus.

The comparative analysis of the effects of practicing physical exercise versus adopting a sedentary lifestyle in the prepartum periods related to two different pregnancies of the same person, in the current case, the young woman that was the subject of our study, highlighted that the sedentary lifestyle adopted during the first pregnancy led to the onset of gestational diabetes, while the practice of physical exercise in the prepartum period related to the second pregnancy decreased the risk of recurrence of this condition in the gestational period.

The practice of physical exercise in the prepartum period of the second pregnancy led to a reduction in postural deficits and a decrease in pain caused by lumbar hyper lordosis, even if they had been present during the first gestational period.

Body dimensions assessed after the second pregnancy were significantly improved compared to those assessed in the postpartum period of the first pregnancy due to the practice of physical exercise in the gestational period. 


\section{References}

ACOG. (2020). Physical activity and exercise during pregnancy and the postpartum period: ACOG Committee opinion summary. Obstetrics \& Gynecology, 135(4), 991-993. https://doi.org/10.1097/aog.0000000000003773

Fazzi, C., Saunders, D. H., Linton, K., Norman, J. E., \& Reynolds, R. M. (2017). Sedentary behaviours during pregnancy: A systematic review. International Journal of Behavioral Nutrition and Physical Activity, 14(1), 32. https://doi.org/10.1186/s12966-017-0485-z

Da Silva, S. G., Ricardo, L. I., Evenson, K. R., \& Hallal, P. C. (2017). Leisure-time physical activity in pregnancy and maternal-child health: A systematic review and meta-analysis of randomized controlled trials and cohort studies. Sports Medicine, 47(2), 295-317. https://doi.org/10.1007/s40279-016-0565-2

Davenport, M. H., Kathol, A. J., Mottola, M. F., Skow, R. J., Meah, V. L., Poitras, V. J., Garcia, A. J., Gray, C. E., Barrowman, N., Riske, L., Sobierajski, F., James, M., Nagpal, T., Marchand, A. A., Slater, L. G., Adamo, K. B., Davies, G. A., Barakat, R., \& Ruchat, S.-M. (2019). Prenatal exercise is not associated with fetal mortality: A systematic review and meta-analysis. British Journal of Sports Medicine, 53(2), 108-115. https://doi.org/10.1136/bjsports-2018-099773

Davies, G., \& Artal, R. (2018). It's time to treat exercise in pregnancy as therapy. British Journal of Sports Medicine, 53(2), 81. https://doi.org/10.1136/bjsports-2018-100360

Dipietro, L., Evenson, K. R., Bloodgood, B., Sprow, K., Troiano, R. P., Piercy, K. L., VauxBjerke, A., \& Powell, K. E. (2019). Benefits of physical activity during pregnancy and postpartum. Medicine \& Science in Sports \& Exercise, 51(6), 1292-1302. https://doi.org/10.1249/mss.0000000000001941

Evenson, K. R., Barakat, R., Brown, W. J., Dargent-Molina, P., Haruna, M., Mikkelsen, E. M., Mottola, M., Owe, K. M., Rousham, E. K., \& Yeo, S. (2014). Guidelines for physical activity during pregnancy: Comparisons from around the world. American Journal of Lifestyle Medicine, 8(2), 102-121. https://dx.doi.org/10.1177\%2F1559827613498204

Gutke, A., Östgaard, H. C., \& Öberg, B. (2006). Pelvic girdle pain and lumbar pain in pregnancy: A cohort study of the consequences in terms of health and functioning. Spine, 31(5), 149-155. https://doi.org/10.1097/01.brs.0000201259.63363.e1

Hytten, F. E. (1968). Physiological changes in early pregnancy. BJOG: An International Journal of Obstetrics and Gynaecology, 75(12), 1193-1197. https://doi.org/10.1111/j.1471-0528.1968.tb02915.x

Ireland, M. L., \& Ott, S. M. (2000). The effects of pregnancy on the musculoskeletal system. Clinical Orthopaedics and Related Research, 372, 169-179. https://doi.org/10.1097/00003086-200003000-00019

Sinclair, I., St-Pierre, M., Elgbeili, G., Bernard, P., Vaillancourt, C., Gagnon, S., \& Dancause, K. S. (2019). Psychosocial stress, sedentary behavior, and physical activity during pregnancy among Canadian women: Relationships in a diverse cohort and a nationwide sample. International Journal of Environmental Research and Public Health, 16(24): 5150. https://doi.org/10.3390/ijerph16245150 